\title{
APPLICATION OF RSA PRINCIPLES TO IMPROVE THE BIM IN THE ROAD DESIGN PROCESS WITH FOCUS ON THE ROAD RESTRAINT SYSTEMS
}

\author{
J. Nováček ${ }^{1 *}$, T. Kohout ${ }^{1}$, P. Vrtal ${ }^{1}$, Z. Svatý ${ }^{1}$, K. Kocián ${ }^{1}$ \\ ${ }^{1}$ Czech Technical University in Prague, Faculty of Transportation Sciences, Department of Forensic Experts in Transportation, \\ Czech Republic - (jakub.novacek, kohout, vrtalpav, svaty, kocian)@fd.cvut.cz
}

\author{
Commission V, WG V/7
}

KEY WORDS: BIM, Data Administration, Road Safety Audit, Road Safety Management, Restraint Systems, Guardrails.

\begin{abstract}
:
Sustainability is one of the main scientific and public topics of today. This includes the sustainable transport and infrastructure. One of the ways how to achieve higher level of sustainability is through the implementation of BIM (Building Information Modelling) into the road design process. However, to create a safe road network, which is also necessary for achievable sustainability, it is essential to consider the Road Safety Audit (RSA) principles throughout the BIM applications. The paper discusses the possibilities of involving the RSA in the process road design in I-BIM (Infrastructures Building Information Modelling). Within the individual phases of the preparation of a transport project and the application of the safety audit tools in BIM applications, it is possible to achieve a significant increase in the level of safety. This approach is shown within the paper on the implementation and use of supplementary road safety devices, specifically the road restraint systems. Currently, the individual components are defined by their main parameters in specific libraries. However, their definition is only as a general object where many of the supplementary parameters or fundamental design characteristics, such as working width or minimal length, are not included. This may lead to potential design flaws or safety hazards which have to be subsequently corrected in further phases. The objective of the paper is to identify the current deficiencies, primarily the missing road safety perspective within the BIM process. The aim is to enable appropriate selection of safety equipment during the design or to provide sufficient information for the mandatory RSA.
\end{abstract}

\section{INTRODUCTION}

BIM or Building Information Model is a modern methodological approach to manage, effectively interpret and archive information about a building project in all phases of its development, including the subsequent management of the final building until the end of its functional cycle. This approach results in the creation of an open database of information about a specific building, which can be viewed by different parts of the professional public involved in the construction or supervision of the building throughout its life cycle (Eastman et. al., 2008).

The of the history of BIM dates back to the 1970s when Charles M. Eastman of the Institute of Physical Planning, CarnegieMellon Univ., Pittsburgh, United States, who defined the principles of the building information model (Eastman et. al., 1974). However, the most significant development of BIM has been reached during the last decade, when BIM was implemented in the current European legislation (Official Journal of the EU,2014). Currently, the use of BIM as the main visualization tool in construction process is not fully adopted in the Czech Republic, but the domestic professional community sees the advantages of switching from pure CAD to BIM. Furthermore, many designers are already using BIM ready software platforms, which will make the transition much easier in the future. BIM platforms are based on components, i.e. on stored parametric elements in object libraries. In general, these stored parameters are in a form of reusable objects that may contain geometric, semantic or topological attributes (Biancardo, et. al., 2020) and (Dell'Acqua, et. al. 2018).
Users of existing graphics programs developed by companies such as Bentley Systems or Autodesk already utilise supplementary libraries which contain the necessary level of parameterization of individual structural elements and thus enable gradual transition to new solutions that are compatible with BIM libraries. In parallel with BIM, the Buildingsmart initiative has been established, specifying the BIM processes and standardizing the format of the data transferred between the different design software, thus enabling greater compatibility between them. This format is called IFC and is supported by a variety of design software. It is essential to understand that BIM is not just a $3 \mathrm{D}$ model of an object with added features and functions. In fact, the primary benefit of BIM is in the management of information. The more valid information the database contains, the more efficient the work will be in the different analyses related to the construction across its life cycle. At the same time, much more informed decisions can be made based on this data. 3D modelling is only one way of representing this information, such as the specification of materials used, certification records or maintenance procedures, or structural and functional parameters within the models (Svatý et. al.,2019).

In parallel with the increasing knowledge of the professional public and the legislative requirements for the adoption of BIM, the requirements for project documentation are also expanding. The construction documents include the position and shape of all structures, which, along with the fact that all the individual components are described and specified, means a significant advance in the detail of the documentation. In general, the level of detail (LOD) of a project must be represented in BIM to determine the level of certainty and accuracy of information contained in the modelled element and to predict the specific 
use of the current information base of a particular project (Pezeshki, et. al. 2018) and (Azhar, 2011). A key aspect is the interactive cooperation between the different experts involved in all phases of the project life cycle in order to input, retrieve or update information in the model. In this iterative process, it is possible to recreate a single virtual model of the design work that is not a mere three-dimensional geometric representation but a "dynamic" model, i.e. it contains a range of additional or complementary information relating to materials, dimensional elements, thermal and energy performance, cost, safety, maintenance, demolition and decommissioning (Biancardo, et. al., 2020).

This article discusses some of the current issues, opportunities and limitations of applying the BIM process to transport infrastructure, particularly when modelling specific elements such as the guardrails and the retaining walls that are not yet well implemented, described, structured or editable in the default BIM-based tool library. At the same time, the paper shows the current shortcomings of the BIM process in the area of the inspection or assessment of socially significant structures (such as linear ground structures) by professional supervisors, i.e. road safety auditors. Specifically, it concerns the process of evaluation of specific construction elements within the individual cycles of its implementation. It is these assessments that should be considered as part of the BIM processes and the conclusions resulting from them should be incorporated into the BIM information database of the specific construction.

\section{THE CURRENT APPROACH}

The topic of applying BIM technology has been prevalent in the construction industry for several decades, however, it is just beginning within the field of transportation construction and infrastructure. The idea of the necessity of implementing BIM technology in the transport sector is outlined in the article "New Perspectives for BIM Usage in Transportation Infrastructure Projects" where a rational analysis of the possible use of BIM in transport infrastructure is presented. In the synthesis of the conclusions of the reviewed studies, it shows that it is possible to apply BIM to existing transport infrastructure (with the use of appropriate software) without transition experience. Finally, the issue of processing and transferring information in BIM between different programs is pointed out, where there was a partial loss of information in the data structure (Moreno 2020), (Wan, et. al., 2019) and (Zou, et. al., 2019).

An already practical example of the use of the BIM process in practice is summarized in the article "Building information Modelling (BIM) application for an existing road infrastructure", which describes the approach of implementing I-BIM for the modernisation of the SS245 road in northern Italy. Designing in BIM included 3D terrain modelling from a point cloud, orientation of horizontal and vertical profiles or modification of cross sections, modelling of the embedded tunnel, creation of a roundabout, generation of a 3D parametric model of the entire road and visualization of the infrastructure in a real-world context. The authors of the paper created a special plugin that allowed extrapolation of data directly from the design program for further calculations. This approach confirmed that the I-BIM application is not only an effective tool for optimization and verification of road design against standards before construction, but also for showing how the infrastructure works within the 3D real-world environmental context (Vingali, et. al., 2021).
Alternative article discusses the combination of BIM and the remote sensing of transport infrastructure. Specifically, it discusses the 3D laser scanning of the roadway and its surroundings as a tool for the monitoring of the current conditions. In accordance with IFC 4.1, road irregularities, traffic signs, guardrails, etc. can be detected by an automatic processing of 3D point clouds. This data can then be effectively used within the BIM to manage the existing road network. The information presented in this paper also confirms the necessity of BIM application and indicates the benefits of unifying the BIM process and various forms of modern technological capabilities in the field of remote sensing of transport infrastructure (Justo, et. al., 2021).

The development of linear structures is very time and cost consuming, so various software platforms are currently being used to speed up this activity. These include for example the automatic section creation, cost estimation or construction time planning. Kim et. al. discusses the advantages and possibilities of implementing these applications within BIM. The contribution presents a development of an optimal approach in order to improve the current situation, especially in the area of transport infrastructure using a 3D oriented approach (Kim, et. al., 2014).

However, the BIM methodological approach can also be utilised on the already constructed and operated transport infrastructure. As an example, the bridge structures can be use due to their rather specific character in comparison to other road structures. By maintaining bridges using BIM, it is possible to optimise the financial resources for maintenance and also ensure its adequate condition with regard to the operational safety. The application of BIM to the historic Izbor bridge in Spain is discussed by León-Robles, which proposes a specific procedure for a particular type of bridge. Subsequently, the bridge BIM model can be generalised and used as a reference model for similar bridge structures (León-Robles, et. al., 2019).

On the other hand, the BIM processes currently faces a number of software challenges. Biancardo presented the real application of BIM in the construction preparation. In the context of design preparations, the authors encountered a problem with interoperability of BIM-based tools and outputs between different programs or platforms. In particular, they focused on the problem with the Road Restraint Systems libraries. They described the lack of standardisation and necessary definitions which led to the loss of information and suggested a way how to improve this situation (Biancardo, et. al, 2020).

After an overall assessment of the available scientific publications, it can be stated that the issue of implementation of the knowledge from the safety audit in the field of passive safety of roads into the BIM process has not been sufficiently pointed out and this issue is not sufficiently accented. At the same time, it is evident that the issue of the road user safety within BIM is not given as much attention as to other aspects. Not only it is significant flaw due to its importance to the road design principles, but it also is not in line with EU transport policy and strategic documents dedicated to this issue. Therefore, in order to achieve a socially acceptable level of transport safety and to move towards the fulfilment of the premise of Vision Zero (White Paper, 2011), it is essential to pay attention to the implementation of road user safety issues and currently used safety practises within the BIM process. 


\section{SAFETY ASSESSMENT OF THE ROAD NETWORK}

In the case of safety assessment of transport structures, two types of approach are considered to improve transport safety. These are reactive and proactive approaches. The former approach relies on the analysis of available data on previously occurring traffic accidents, while the second approach focuses on the analysis of the construction and operational characteristics of existing roads in order to identify current and future safety deficiencies (Road Safety Manual, 2004). The objective is to identify the problematic or risky locations or the construction deficiencies, ideally in advance, and then implement appropriate countermeasures to ensure the sufficient safety of the infrastructure. In this way, the potential deficiencies can be identified during the pre-construction phases. Furthermore, thanks to the complete data model contained in BIM, all construction details could be assessed already at the design phase. Therefore, it is not necessary to correct them during the construction work where it leads to significant increase of costs.

The safety assessment in the context of transport projects presents a rather complicated matter and differs significantly from the assessment of other types of projects, such as buildings. A wide range of various information and parameters affect and has to be considered within the assessment. It is influenced not only by the operational or spatial characteristics of the transport infrastructure itself, but also by the psychology of drivers, the traffic volumes or other aspects. It is also important to evaluate the particular road section with consideration to the adjacent road sections that are not primarily assessed in terms of the project, but are closely related to the project. Yet, it is not entirely possible to implement all of these elements in the BIM models themselves, which may be one of the reasons why they are currently being omitted and are not given the importance that would be desirable from the point of view of a socially acceptable level of the transport safety. (Svatý et. al.,2019). Nevertheless, there is an opportunity to use existing databases containing information that can effectively complement BIM models. Possible examples of useful databases available in the Czech Republic include the "National Transport Census" portal, traffic accident statistics or the "Central Register of Road Safety Analysis" (CEBASS, 2016) or similar knowledge databases.

Similar sources can be found in all developed countries and thus, the principles can be utilised universally. The implementation of these data into the BIM model would lead to a higher predictive value of non-graphical data, easier evaluation of the predictive level of safety and improving the construction management strategy of the responsible institutions.

This approach could improve the assessment and control of the road construction from the perspective of road safety auditors in particular, but the usefulness of this additional information could certainly also serve road managers who could draw on and use all the information about the road in future years. By compiling and archiving these datasets, all aspects of a newly designed or reconstructed road could be assessed in aggregate form and there would be no risk that any information could be missed or not available.

\section{ROAD RESTRAINT SYSTEMS}

One of the main elements of the passive safety of any road are the road restraint systems. Their primary function is to restrain vehicles so that they do not run off the road as a result of an emergency, thus decreasing the consequences of an accident. This is one of the key elements that is commonly used and their proper placement and utilisation is often addressed in the RSA. Their installation is dependent on several factors that directly affect the functionality of the restraint system itself. However, in the current BIM templates, these devices are not parameterised in sufficient level of detail and both their accurate 3D visualization and precise specification of their functional characteristics are not available.

The impossibility of proper use of parametric blocks is also discussed in "BIM-Based Design for Road Infrastructure: A Critical Focus on Modeling Guardrails and Retaining Walls", which also mentions the issue and discusses the shortcomings associated with modelling retaining facilities. The problems consist mainly in the lack of types and kinds of individual guardrails and the absence of detailed parameters for each component. At the same time, special parts of the guardrails are not completed, for example, leading or trailing parts, connections between types or different forms of guardrails termination, e.g. in the form of a terminal (Biancardo, et. al., 2020).

The problem with the construction deficiencies is the lack of quality of the graphic design. The current work is mainly based on combination of different graphic software (Civil 3D, Revit) and only afterwards implementation of resulting outputs into the BIM databases. For example, the BIM-tool Civil 3D does not allow to parameterize elements with additional information that is directly attached to the model, such as manufacturer's technical data sheets or other parameters and information important for both project development and management, and it is necessary to modify it further in other programs. Another example shows that the way they are implemented is still insufficient, as the individual model blocks are not well enough connected to each other. At the same time, even in their current design, they do not allow, for example, to faithfully reproduce the appropriate geometric position of the guardrails in the terrain. The biggest deficiencies are currently observed in the locations of the apex curves, where it is problematic to connect the individual blocks well enough to make the whole model look realistic. This may seem as unnecessary; however, it can be used to a great advantage for the purposes of the evaluation of the driver sight conditions.

Within the professional studies conducted at the [names and affiliation removed for double-blind review] a project focusing on the analysis of individual types of the most commonly used guardrails in the Czech Republic was created. One of the aims was to summarise and present necessary information about various types of guardrails with considerations to the potential use within BIM libraries. The outputs were subsequently also presented in an unified form of catalogue sheets, where each specific type of guardrail has its main parameters defined. The parameters include the main constructional characteristics, placement restrictions and are also supplemented with a crosssection and photographic documentation (see fig. 1). The classification was carried out for guardrails commonly used on the corridors of the Trans-European Transport Network (TEN-T). A total of $4471 \mathrm{~km}$ of motorways and class I roads were analysed, in which a total of 47 types of guardrails were identified.

The following figure illustrate the layout of the catalogue sheet for a specific type of safety barriers. In the upper part is located a photographic documentation of the selected guardrail. Below 
is a schematic layout and a cross-section of the barrier. The bottom part contains basic design parameters of selected guardrail.

TPV 167/2015
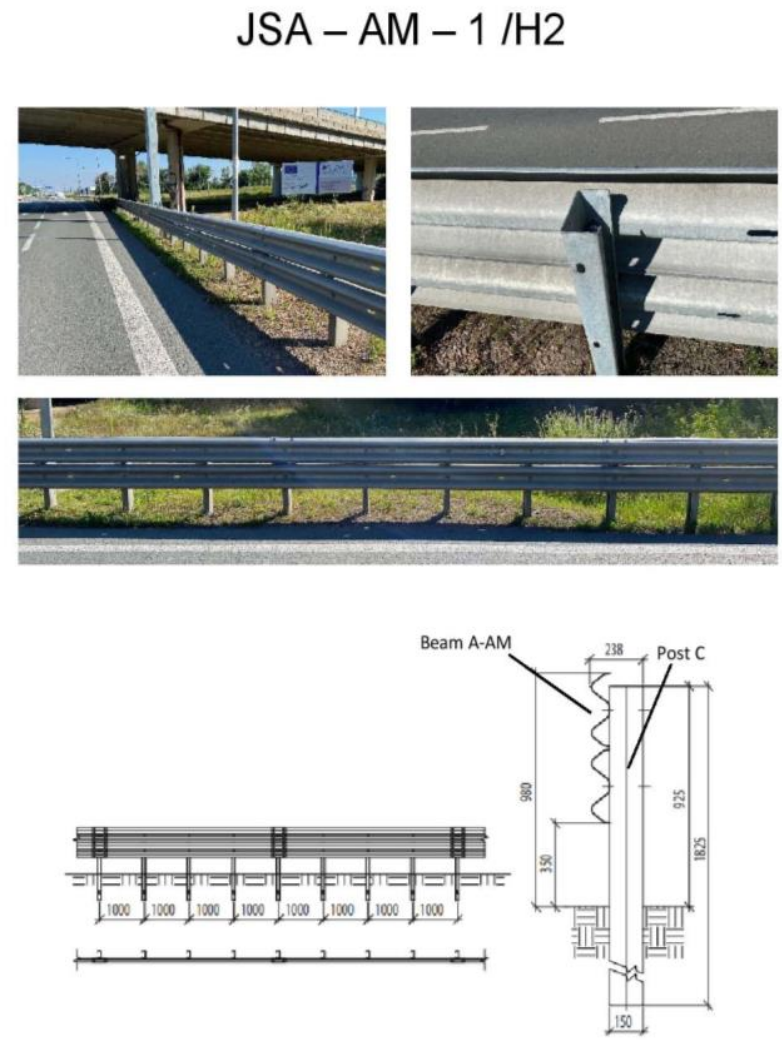

Guardrail parameters

\begin{tabular}{|c|c|c|}
\hline \multicolumn{2}{|l|}{ Level of restraint } & $\mathrm{H} 2$ \\
\hline \multicolumn{2}{|l|}{ Working (effective) width [m] } & $1,1(\mathrm{~W} 4)$ \\
\hline \multirow{2}{*}{\multicolumn{2}{|c|}{$\begin{array}{l}\text { Face of guardrail to obstacle distance [m] } \\
\text { Guardrail height [m] }\end{array}$}} & 1,1 \\
\hline & & 0,980 \\
\hline \multicolumn{2}{|c|}{ Guardrail height [m] } & 40 \\
\hline & $>80[\mathrm{~km} / \mathrm{h}]$ & 60 \\
\hline
\end{tabular}

Figure 1. Example of the catalogue sheet for guardrail JSA - AM $-1 / \mathrm{H} 2$.

\section{Guardrail design parameters classification}

The main design parameters of the guardrails are defined by five main parameter groups. These are the level of restraint of the guardrail, the working width of the guardrail, the minimum length in front of and behind the obstacle, the height of the guardrail and the minimum length of the guardrail. To give an example of the parameter groups, three were selected and are further described. The presented definitions are related to the Czech Republic; however, the general demands are similar within all European Union with slight deviations.

In the Czech Republic, the level of restraint is defined in technical conditions No. 114 issued by the Ministry of Transport. The general definition is that the level of restraint is "The verified value of the side impact by the vehicle which the guardrail is capable of resisting without being overcome by the vehicle, while ensuring the required value of impact severity and acceptability of the guardrail behaviour". A test based on the crash tests or numerical simulations has to be carried out to determine or classify the barrier according to the level of restraint (Ministry of Transport, Technical Conditions no. 114, 2020).

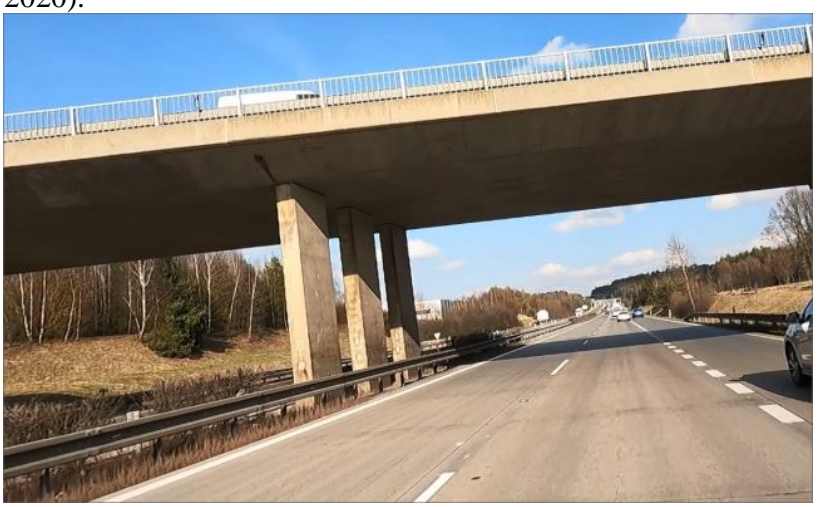

Figure 2. Identified insufficient levels of restraint during RSA on the D5 motorway (Prague - Nuremberg)

The working width is another basic parameter determining the characteristics of the guardrail. Specifically, this parameter represents "The distance between the face of the barrier before impact and the maximum dynamic position of any major part of the restraint system". For each type of guardrail there is a different working width, which is determined by impact tests. Subsequently, the distance of the fixed obstacle from the face of the guardrail that will be in accordance with the test results shall be determined on the basis of the impact test (ČSN EN 1317-2, 2011).

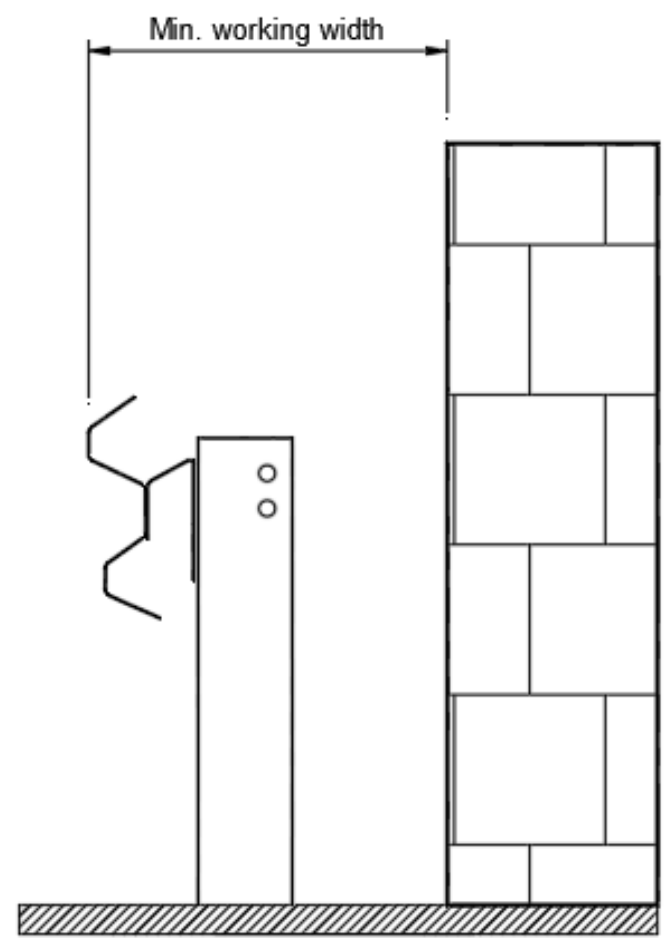

Figure 3. Minimum working width between face of the guardrail and protected obstacle.

The minimum length of need of the guardrail shall be determined for steel guardrails on the basis of the results of impact or simulation tests. If the maximum permissible speed is above $80 \mathrm{~km} / \mathrm{h}$, then the length of the railing shall be the same as the results of the impact tests. In the case of speeds below 80 $\mathrm{km} / \mathrm{h}$, the total length of the guardrail may be reduced to up to 
$70 \%$ of the recommended length by the impact test (Ministry of Transport, Technical Conditions No. 114, 2020).

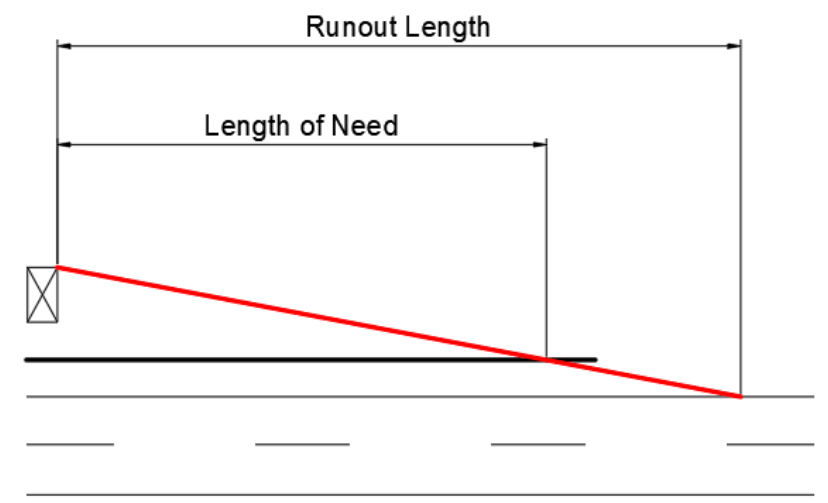

Figure 4. Minimum length of need for guardrail.

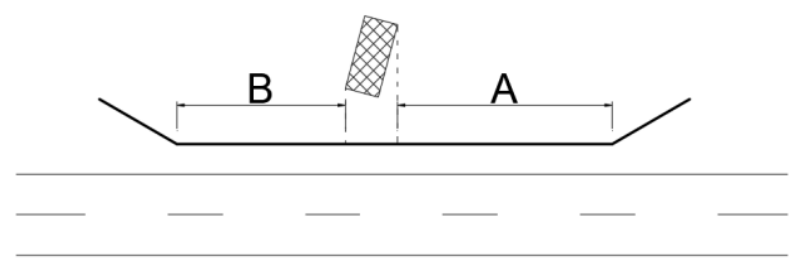

Figure 5. Minimum length of guardrail in front of (A) and behind (B) protected obstacle.

The resulting database of the most frequently occurring guardrails can serve as a supporting tool for implementation and utilisation of guardrails in the BIM workflow. Furthermore, graphical models can be also constructed according to this database. If necessary, the database can be extended with additional parameters e.g. manufacturer's brand, price, weight, etc. However, the five main parameter groups were selected with an aim to cover all the most important parameters which are necessary to enable the assessment of the appropriate functionality of the restraint in relation to the specific location of the installation or the specific road. Another important aspect is that different characteristics are required from road restraint systems on motorways, and different on roads of lower traffic significance. With the implementation of sufficiently detailed restraint system library, it would be possible to utilise this information during the design phase. The user could select the most suitable type of guardrail according to certain parameters and subsequently determine the actual restraint system through already prefiltered database options. At the same time, the catalogue of guardrails could be extended with information if the specific guardrails have been already used on a specific road (or certain road type) or have been already approved through the RSA at similar road. This additional information value, indicating the suitability of the use of a given type of guardrail, would again simplify the process of designing guardrails within the BIM process and make it more efficient and safer.

Additionally, the [names and affiliation removed for doubleblind review] is currently trying to further extend the available catalogue sheets and validate all of the selected parameters in cooperation with the Road and Motorway Directorate of the Czech Republic. The preliminary results clearly shows a high potential for utilisation and practical implementation within the BIM process.

\section{CONCLUSION}

Transport infrastructure is an indicator of the economic and social level of any country. However, the road infrastructure is associated with significant costs throughout its life cycle and requires efficient management in all of its phases. Current management and control systems are inefficient due to the extensive quantity of inconsistent information. The opportunity in this aspect is the transformation to modern and automatic management. The use of BIM for infrastructure management can help to make infrastructure more reliable, sustainable and safer, while reducing maintenance costs and the level of risk in the implementation and operation of construction. The development of BIM modelling presents a key element for future data handling, maintenance and detailed visualization of any structure under construction, not just transport ones. It is clear that there are still a number of conceptual deficiencies in this area and further work is necessary. The use of BIM within the design documentation of transport line structures for subsequent control by RSA auditors seems to be necessary in the future in order to further increase the level of safety of transport structures and to clearly summarize all available data. Currently, the primary weakness can be identified as the limited consideration towards the transport safety. It is primarily about implementation of the current level of knowledge in this area into the process of design phases of a specific linear construction in the BIM process. The primary objective should be to complete the parameterisation of the individual elements contributing to road safety. This contribution presented this idea through the restraint systems and discusses the possibility of effective implementation of the current knowledge. However, other elements that reduce the secondary risk arising from a traffic accident, such as embankment or shock absorbers (attenuators), should also be included. In the authors' opinion, a similar methodological approach with only slight adjustments can be applied to this road equipment. The above efforts are fully in line with the current European transport policy and the ideas of Vision Zero to create a socially acceptable level of road safety.

\section{ACKNOWLEDGEMENTS}

This work was supported by the Grant Agency of the Czech Technical University in Prague, grant No. SGS20/199/OHK2/3T/16.

\section{REFERENCES}

Azhar, S., 2011: Building information modeling (BIM): Trends, benefits, risks, and challenges for the AEC industry. Leadersh. Manag. Eng. 2011, 11, 241-252.

Biancardo, S.A.; Capano, A.; Oliveira, S.G.; Tibaut, A., 2020: Integration of BIM and Procedural Modeling Tools for Road Design. Infrastructures 2020, 5, 37.

Biancardo, S.A.; Viscione, N.; Cerbone, A.; Dessì, E., Jr. BIMBased Design for Road Infrastructure: A Critical Focus on Modeling Guardrails and Retaining Walls. Infrastructures 2020, 5, 59. https://doi.org/10.3390/infrastructures5070059.

CEBASS, 2016: Central Register of Road Safety Analysis, CTU in Prague, Faculty of Transportation Sciences, Available from: https://cebass.rsd.cz/. 
Czech Technical Standard, ČSN EN 1317-2, Road restraint systems - Part 2: Performance classes, impact test acceptance criteria and test methods for safety barriers including vehicle parapets, 2011.

Dell'Acqua, G.; De Oliveira, S.G.; Biancardo, S.A., 2018: Railway-BIM: Analytical review, data standard and overall perspective. Ing. Ferrov. 2018, 73, 901-923.

Eastman, C., Fisher, D., Laufe, G., Lividini, J., Stoker, D., Yessios, C., 1974: An Outline of the Building Description System., Institute of Physical Planning, Carnegie-Mellon University.

Eastman, C., Teicholz, P., Sacks, R., 2008: BIM Handbook. Hoboken, NJ, Wiley, ISBN 978-0470185285.

European Comission, 2011: White Paper - Roadmap to a single European transport area - Towards a competitive and resourceefficient transport system, European Comission, Luxembourg, ISBN 978-92-79-18270-9.

Justo, A., Soilán, M., Sánchez-Rodríguez, A., Riveiro, B., 2021:Scan-to-BIM for the infrastructure domain: Generation of IFC-compliant models of road infrastructure assets and semantics using 3D point cloud data, Automation in Construction, Volume 127, 2021, 103703, ISSN 0926-5805, https://doi.org/10.1016/j.autcon.2021.103703.

Kim, H., Orr, K., Shen, Z., Moon, H., Ju, K., Choi, W.,2014: Highway Alignment Construction Comparison Using ObjectOriented 3D Visualization Modeling, Journal of Construction Engineering and Management, Volume 140, Number 10, 05014008, 2014 https://ascelibrary.org/doi/abs/10.1061/\%28ASCE\%29CO.1943 $-7862.0000898$.

León-Robles, C.A.; Reinoso-Gordo, J.F.; González-Quiñones, J.J. Heritage Building Information Modeling (H-BIM) Applied to A Stone Bridge. ISPRS Int. J. Geo-Inf. 2019, 8, 121. https://doi.org/10.3390/ijgi8030121.

Ministry of Transport, 2020: Technical Conditions no.114, Crash Barriers on Roads.

Moreno Bazán, Á.; Alberti, M.G.; Arcos Álvarez, A.; Trigueros, J.A. New Perspectives for BIM Usage in Transportation Infrastructure Projects. Appl. Sci. 2020, 10, 7072. https://doi.org/10.3390/app10207072.

Official Journal of the EU,2014: Directive 2014/24/EU of the European Parliament and of the Council of 26 February 2014 on public procurement and repealing Directive 2004/18/EC Text with EEA relevance, ELI: http://data.europa.eu/eli/dir/2014/24/oj.

Pezeshki, Z.; Ivari, S.A.S., 2018: Applications of BIM:ABrief Review and Future Outline. Arch. Comput. Methods Eng. 2018, $25,273-312$

Road Safety Manual, 2004:, Recommendations from the World Road Association (PIARC), PIARC Technical Committee on Road Safety (C13).

Svatý, Z., Kocián, K., Mičunek, T., 2019: Integration of Safety Assessment in BIM for Transportation Infrastructure., CTU in
Prague, Faculty of Transportation Sciences, Department of Forensic Experts in Transportation.

Vingali, V., Acerra, E.M., Lantieri, C., Di Vincenzo, F., Piacentini, G., Pancaldi, S., 2021: Building information Modelling (BIM) application for an existing road infrastructure, Automation in Construction, Volume 128, 2021, 103752, ISSN 0926-5805, https://doi.org/10.1016/j.autcon.2021.103752.

Wan, C.; Zhou, Z.; Li, S.; Ding, Y.; Xu, Z.; Yang, Z.; Xia, Y.; Yin, F. Development of a Bridge Management System Based on the Building Information Modeling Technology. Sustainability 2019, 11, 4583. https://doi.org/10.3390/su11174583.

Zou, Y., Kiviniemi, A., Jones, S.W. et al. Risk Information Management for Bridges by Integrating Risk Breakdown Structure into 3D/4D BIM. KSCE J Civ Eng 23, 467-480 (2019). https://doi.org/10.1007/s12205-018-1924-3. 